\title{
Multidrug resistance-associated protein 2 determines the efficacy of cisplatin in patients with hepatocellular carcinoma
}

\author{
PAVEL V. KORITA ${ }^{1,2}$, TOSHIFUMI WAKAI ${ }^{2}$, YOSHIO SHIRAI ${ }^{2}$, YASUNOBU MATSUDA $^{3}$, \\ JUN SAKATA ${ }^{2}$, MASAAKI TAKAMURA ${ }^{3}$, MASAHIKO YANO ${ }^{3}$, AYUMI SANPEI ${ }^{2}$, \\ YUTAKA AOYAGI ${ }^{3}$, KATSUYOSHI HATAKEYAMA ${ }^{2}$ and YOICHI AJIOKA ${ }^{1}$ \\ ${ }^{1}$ Division of Molecular and Diagnostic Pathology, ${ }^{2}$ Division of Digestive and General Surgery, and \\ ${ }^{3}$ Division of Gastroenterology and Hepatology, Niigata University Graduate School of Medical \\ and Dental Sciences, 1-757 Asahimachi-dori, Chuo-ku, Niigata 951-8510, Japan
}

Received December 2, 2009; Accepted January 22, 2010

DOI: 10.3892/or_00000721

\begin{abstract}
We hypothesized that expression of multidrug resistance-associated protein 2 (MRP2), a major cisplatin transporter, may determine the efficacy of cisplatin as a treatment for patients with hepatocellular carcinoma (HCC). A prospective analysis was conducted of 49 consecutive patients who underwent resection for HCC (16 patients treated with cisplatin-based neoadjuvant chemotherapy and 33 patients treated without neoadjuvant chemotherapy). Expression of MRP2 in resected specimens was assessed by immunohistochemical and Western blot analyses. The extent of tumor necrosis was assessed histologically in the greatest dimension of the tumor specimen from each patient. The median percentage of tumor necrosis was $81 \%$ (range: $0-100 \%$ ) and complete tumor necrosis was found in 3 patients. Overexpression of MRP2 was detected in 24/46 (52\%) tumor specimens. In 16 patients treated with cisplatin, tumor size and dose of cisplatin did not correlate with tumor necrosis of the resected specimens $(\mathrm{P}=0.706$ and $\mathrm{P}=0.555$, respectively). Of 13 tumor specimens containing vivid tumor from 16 patients treated with cisplatin, 8 had overexpression of MRP2. Tumor specimens with overexpression of MRP2 showed a lower percentage of tumor necrosis than those with non-overexpression (median percentage of tumor necrosis, $19 \%$ vs. $99 \%, \mathrm{P}=0.003)$. In conclusion, overexpression of MRP2 correlates with a lower percentage of tumor necrosis in patients treated with cisplatin-based neoadjuvant chemotherapy for $\mathrm{HCC}$, whereas either tumor size or dose of cisplatin does
\end{abstract}

Correspondence to: Dr Toshifumi Wakai, Division of Digestive and General Surgery, Niigata University Graduate School of Medical and Dental Sciences, 1-757 Asahimachi-dori, Chuo-ku, Niigata 951-8510, Japan

E-mail: wakait@med.niigata-u.ac.jp

Key words: hepatocellular carcinoma, drug resistance, multidrug resistance-associated protein 2 , chemotherapy, cisplatin not. Expression of MRP2 determines the efficacy of cisplatinbased chemotherapy in patients with HCC.

\section{Introduction}

Multidrug resistance-associated protein 2 (MRP2; ABCC2), formally known as ATP-binding cassette (ABC), sub-family $\mathrm{C}$, member 2, is a member of the superfamily of $A B C$ transporters (1). MRP2 is localized to the canalicular (apical) membrane of hepatocytes (2-4), where it functions as a major exporter of organic anions, drugs, conjugated bilirubin, and bile salts to bile canaliculi (2-6).

MRP2 is one of the major transporters of cisplatin (7-9). In vitro experiments have shown that elevated expression of MRP2 decreases cisplatin accumulation in HCC cells and contributes to cisplatin resistance (9). Transfection of MRP2 antisense cDNA into a human hepatoma cell line decreased the MRP2 protein level and increased sensitivity to cisplatin (10). MRP2 expression in resected tumor specimens of patients with HCC, as detected by immunohistochemical analysis, ranges from 63 to $90 \%$ (11-13). In addition, MRP2 expression in tumor specimens is increased compared to non-neoplastic liver tissues using quantitative RT-PCR and Western blot analyses $(13,14)$. The effect of tumor expression of MRP2 on the efficacy of cisplatin administration for patients with HCC has not been investigated previously. The aim of the current study was to test the hypothesis that expression of MRP2 may determine the efficacy of cisplatin-based neoadjuvant chemotherapy for patients with HCC.

\section{Materials and methods}

Patient population. From March 2007 through December 2008, a total of 59 consecutive Japanese patients with resectable HCC were referred to the Division of Digestive and General Surgery, Niigata University Medical and Dental Hospital (Niigata, Japan). Ten patients who received ablation therapy prior to surgical resection were excluded. The remaining 49 patients formed the basis of this prospective pilot study and included 39 men and 10 women with a median age of 70 years (range: $40-81$ years). Signed informed consent 
to participate in the current study was obtained from all patients. The current study was approved by the Institutional Review Board of Niigata University Medical and Dental Hospital.

Neoadjuvant chemotherapy. During the study period, neoadjuvant chemotherapy was applied to prevent tumor progression when a patient was on the waiting list for definitive operation for HCC for more than one month. The decision to use neoadjuvant chemotherapy was made by the Institutional Cancer Committee of Niigata University Medical and Dental Hospital. Indications for neoadjuvant chemotherapy included multiple hepatic tumors or a solitary tumor $>3 \mathrm{~cm}$ in diameter, because these preoperative factors are closely associated with vascular invasion or poor post-resection survival (15-17). In the current series, 16 patients received neoadjuvant chemotherapy, which consisted of hepatic arterial infusion of a fine-powder formulation of cisplatin (IA-call ${ }^{\circledR}$, Nippon Kayaku, Co., Ltd., Tokyo, Japan; recommended dose of $65 \mathrm{mg} / \mathrm{m}^{2}$ ) under the guidance of hepatic angiography. The remaining 33 patients did not undergo neoadjuvant chemotherapy for HCC. The size of the largest hepatic tumor ranged from 1.5 to $12.1 \mathrm{~cm}$ (median tumor size, $3.5 \mathrm{~cm}$ ) on contrast-enhanced spiral computed tomography (CT) images before neoadjuvant chemotherapy.

The response to neoadjuvant chemotherapy was assessed by contrast-enhanced spiral CT and was evaluated according to the Response Evaluation Criteria in Solid Tumors (RECIST) guidelines (18). Treatment-related toxicity was evaluated according to the Common Terminology Criteria of Adverse Events (CTCAE version 4.0; National Cancer Institute, Bethesda, MD, USA) (19).

Hepatectomy procedures. A hepatectomy procedure was selected for each patient, taking the primary tumor status (size, number, location), the hepatic functional reserve, and the patient's general condition into account (16). In the current study, the term 'major hepatectomy' indicated formal hemihepatectomy or more extensive resection, whereas less extensive hemihepatectomy was designated 'minor hepatectomy'. Postoperative morbidity was defined as any postoperative complication that lengthened the hospital stay (16).

Pathologic evaluation. Resected specimens were submitted to the Department of Surgical Pathology of Niigata University Medical and Dental Hospital. Each specimen was examined to determine the presence of cirrhosis, the number of hepatic tumors, the size of the largest hepatic tumor, the histologic grade, and gross or microscopic vascular invasion. The pathologic findings were described according to the TNM Classification of Malignant Tumours by the International Union Against Cancer (6th edition, 2002) (20).

A total of 90 hepatic tumors were resected in the current series. Twenty-eight patients had a solitary tumor and 21 had multiple tumors. The number of hepatic tumors was determined by gross examination of multiple slices from each resected specimen, but did not include satellite nodules. The definition of satellite nodules followed that of Taylor et al (21), regarding colorectal carcinoma liver metastasis. In

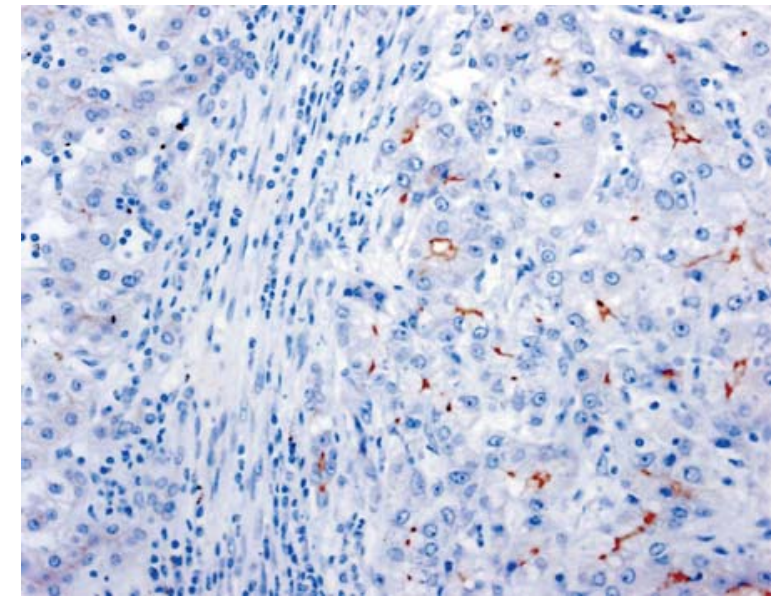

Figure 1. Hepatocellular carcinoma with overexpression of multidrug resistance-associated protein 2 (MRP2). Non-neoplastic hepatocytes in the left half show faint MRP2 expression in the canalicular membranes of hepatocytes, whereas cancerous tissue in the right half has overexpression of MRP2. Immunohistochemical staining: original magnification, x400.

patients with multiple tumors, the largest tumor was chosen as representative of all tumors.

The microscopic diagnosis of cirrhosis in the adjacent non-neoplastic liver was defined as the presence of regenerative nodules surrounded by fibrous septa. Using these criteria 33 patients had liver cirrhosis histologically verified. The median tumor size was $2.8 \mathrm{~cm}$ (range: $1.1-10 \mathrm{~cm}$ ) in the resected specimens and the histologic grade was determined according to the Edmondson-Steiner classification (22), which is based on the areas of the tumor with the highest grade. Vascular invasion was defined as gross or microscopic involvement of the vessels (portal vein or hepatic vein) within the peritumoral liver tissue (17).

The extent of tumor necrosis was assessed histologically in the greatest dimension of the tumor specimen from each patient. The percentage of tumor necrosis was defined as the ratio between total necrotic area and the whole area of the tumor, multiplied by 100 . In the greatest dimension of the tumor specimens from 16 patients who received neoadjuvant chemotherapy, the median necrotic area was $113.4 \mathrm{~mm}^{2}$ (range: 0-2862 $\mathrm{mm}^{2}$ ), whereas the median whole tumor area was $336.5 \mathrm{~mm}^{2}$ (range: $57-5498 \mathrm{~mm}^{2}$ ).

Immunohistochemistry. From each resected specimen, 1 to 3 paraffin-embedded block(s) (median, 2 blocks) were used for immunohistochemistry. Three serial $3-\mu \mathrm{m}$ sections were re-cut and prepared from each block; 1 for hematoxylin and eosin staining, 1 for MRP2-immunohistochemical staining, and 1 as a negative control. Two independent surgical pathologists blinded to the clinical details assessed each section.

The streptavidin-biotin immunoperoxidase method was performed using the Histofine SAB-PO (M) kit (Nichirei Biosciences Inc., Tokyo, Japan). The sections were deparaffinized and rehydrated, then microwaved at $500 \mathrm{~W}$ for 7 cycles of $3 \mathrm{~min}$ in $10 \mathrm{mmol} / \mathrm{l}$ sodium citrate buffer $(\mathrm{pH} 6.0)$ to retrieve antigenic activity. Endogenous peroxidase activity was inhibited by incubation with $0.3 \%$ hydrogen peroxidase in methanol for $20 \mathrm{~min}$. Sections were blocked against non-specific reactions 
Table I. Tumor response in 16 patients treated with cisplatin-based neoadjuvant chemotherapy.

\begin{tabular}{|c|c|c|c|c|c|c|}
\hline & \multicolumn{4}{|c|}{ No. of patients } & \multirow[b]{2}{*}{ Response rate $(\%)$} & \multirow[b]{2}{*}{ P-value } \\
\hline & $\mathrm{CR}$ & PR & $\mathrm{SD}$ & PD & & \\
\hline Total & 0 & 4 & 12 & 0 & 25 & \\
\hline No. of tumors & & & & & & 0.569 \\
\hline Solitary & 0 & 3 & 5 & 0 & 37.5 & \\
\hline Multiple & 0 & 1 & 7 & 0 & 12.5 & \\
\hline Tumor size $(\mathrm{cm})$ & & & & & & $>0.999$ \\
\hline$\leq 3$ & 0 & 2 & 6 & 0 & 25 & \\
\hline$>3$ & 0 & 2 & 6 & 0 & 25 & \\
\hline Stage & & & & & & 0.435 \\
\hline I & 0 & 3 & 4 & 0 & 43 & \\
\hline II & 0 & 1 & 6 & 0 & 14 & \\
\hline III & 0 & 0 & 2 & 0 & 0 & \\
\hline
\end{tabular}

CR, complete response; PR, partial response; $\mathrm{SD}$, stable disease; PD, progressive disease.

with $10 \%$ normal rabbit serum, and were then incubated overnight at $4^{\circ} \mathrm{C}$ with mouse anti-MRP2 monoclonal antibody (clone M2III-6; Monosan, Uden, The Netherlands; dilution at $1: 20)$. Sections were then incubated with biotinylated rabbit anti-mouse immunoglobulin for $30 \mathrm{~min}$ followed by incubation with the streptavidin-peroxidase complex for $10 \mathrm{~min}$. Diaminobenzidine was used as the chromogen, and the sections were counterstained with hematoxylin. Normal mouse immunoglobulin was substituted for the primary antibody as a negative control, whereas the immunoreactivity of adjacent non-neoplastic liver tissue was used as an internal positive control.

Pattern of MRP2 immunohistochemical expression in HCC. MRP2 expression was defined as the immunoreactivity of canalicular (apical) membranes of hepatocytes according to the description of Paulusma et al (4). Non-neoplastic hepatocytes showed weak to moderate intensity of MRP2 expression in the canalicular membrane. Immunoreactivity of MRP2 in tumor specimens was evaluated by comparison with adjacent non-neoplastic hepatocytes and classified into 3 categories: unchanged expression, when immunoreactivity of the tumor specimen was similar to that of non-neoplastic hepatocytes; loss-of-expression, characterized by totally negative immunoreactivity throughout the tumor specimen; and diffuse expression, characterized by strong positive immunoreactivity throughout the tumor specimen (Fig. 1). In the current study, overexpression of MRP2 was defined as diffuse expression, whereas non-overexpression was defined as unchanged or loss-of-expression.

Detection of MRP2 expression by Western blot analysis. Tissue samples were prepared for Western blotting by first snap-freezing and then stored at $-80^{\circ} \mathrm{C}$ until used for analysis. Tissue samples from 3 normal livers obtained at surgery for other conditions were processed for analysis as normal controls. Lysate from tissue samples were obtained by homogenization in the lysis buffer [20 mM Tris-buffered with 4-(2-hydroxyethyl)-1-piperazineethanesulfonic acid (HEPES) $\mathrm{pH} 8.0,2 \mathrm{mM}$ ethylenediaminetetraacetic acid (EDTA), $0.5 \mathrm{M}$ sodium chloride, $0.5 \%$ sodium deoxycholate, $0.5 \%$ Triton X-100, 0.25 M sucrose, $50 \mathrm{mM}$ 2-mercaptoethanol, $250 \mu \mathrm{M}$ phenylmethylsulfonyl fluoride and $1 \mu \mathrm{M}$ pepstatin]. The lysate samples were kept on ice for $30 \mathrm{~min}$, filtered through gauze and precleared by centrifugation at $15,000 \mathrm{rpm}$ for $15 \mathrm{~min}$ at $4^{\circ} \mathrm{C}$. Following protein quantification using the Bradford assay (Bio-Rad Laboratories, Hercules, CA, USA), $50 \mu \mathrm{g}$ aliquots of samples were resolved by sodium dodecyl sulfate-polyacrylamide gel electrophoresis and transferred to Immobilon membranes (Millipore, Bedford, MA, USA). Nonspecific sites binding sites on the membranes were blocked in 5\% skim milk, whereupon filters were incubated with anti-MRP2 antibody (clone M2III-6; Monosan, Uden; dilution at 1:1000) and then the appropriate horseradish peroxidase-conjugated secondary antibodies. After the detection was performed using enhanced chemiluminescence reagent (GE Healthcare, Buckingham, UK), the blots were stripped, washed, and reprobed for $\beta$-actin (Santa Cruz Biotechnology, Santa Cruz, CA, USA; dilution at 1:5000).

Statistical analysis. Medical records were obtained from all 49 patients. Categorical variables were compared by the Fisher exact test or the Pearson $\chi^{2}$ test; continuous variables were compared by the Mann-Whitney U test. Correlation between 2 continuous variables was evaluated by the Spearman rank correlation. All statistical evaluations were performed using the SPSS 16.0J software package (SPSS Japan, Tokyo, Japan). All tests were two-sided and $\mathrm{P}<0.05$ was considered statistically significant.

\section{Results}

Tumor response in 16 patients treated with cisplatin-based neoadjuvant chemotherapy. In 16 patients who received 
Table II. Toxicity in 16 patients treated with cisplatin-based neoadjuvant chemotherapy.

\begin{tabular}{|c|c|c|c|c|}
\hline \multirow[b]{2}{*}{ Characteristics } & \multicolumn{4}{|c|}{ No. of patients } \\
\hline & Grade 1 & Grade 2 & Grade 3 & Grade 4 \\
\hline \multicolumn{5}{|l|}{ Hematological toxicity } \\
\hline Leukocytopenia & 2 & 1 & 0 & 0 \\
\hline Anemia & 0 & 1 & 0 & 0 \\
\hline Thrombocytopenia & 2 & 2 & 0 & 0 \\
\hline \multicolumn{5}{|l|}{ Non-hematological toxicity } \\
\hline Fever & 2 & 0 & 0 & 0 \\
\hline Diarrhea & 1 & 0 & 0 & 0 \\
\hline Decreased albumin level & 2 & 7 & 0 & 0 \\
\hline Elevated total bilirubin level & 4 & 1 & 0 & 0 \\
\hline Elevated AST level & 2 & 8 & 2 & 0 \\
\hline Elevated ALT level & 3 & 7 & 1 & 0 \\
\hline Elevated creatinine level & 6 & 0 & 0 & 0 \\
\hline
\end{tabular}

AST, aspartate aminotransferase; ALT, alanine aminotransferase.

A

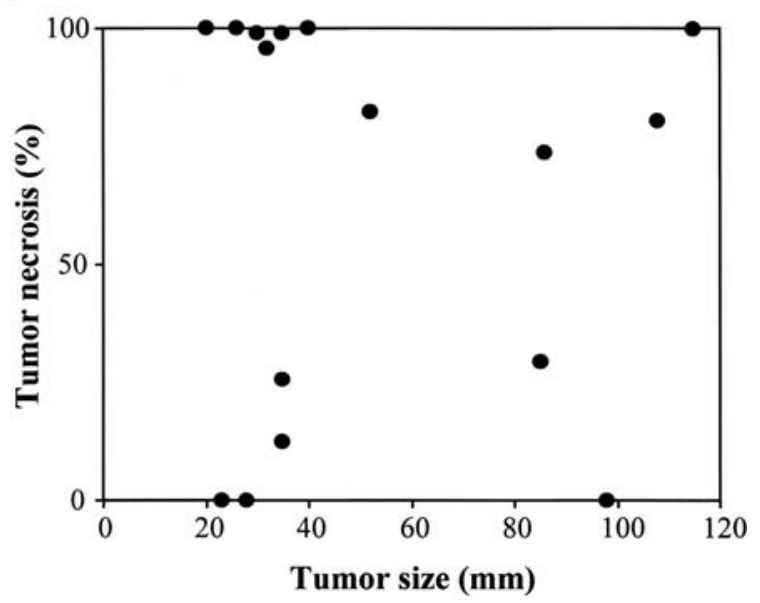

B

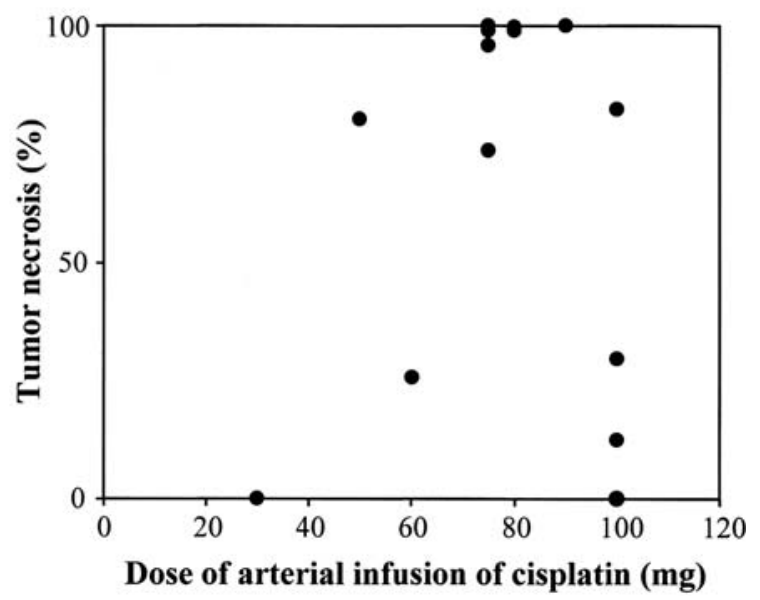

Figure 2. Correlation of tumor necrosis with tumor size and dose of cisplatin. (A) Tumor size prior to neoadjuvant chemotherapy does not correlate with tumor necrosis (correlation coefficient $=-0.102 ; \mathrm{P}=0.706$ ). (B) The dose of hepatic arterial infusion of cisplatin has no correlation with tumor necrosis (correlation coefficient $=-0.160 ; \mathrm{P}=0.555)$.

cisplatin-based neoadjuvant chemotherapy, the median dose of cisplatin was $77.5 \mathrm{mg}$ per body (range: 30-100 mg per body). The overall response rate of these patients was $25 \%$; the therapeutic efficacy according to the RECIST guidelines was not associated with tested tumor-related factors (Table I).

Toxicity in 16 patients treated with cisplatin-based neoadjuvant chemotherapy. All 16 patients were assessed for toxicity and no toxic deaths occurred. The incidences of the main treatmentrelated toxicity according to the CTCAE version 4.0 are listed in Table II as the maximum grade seen per patient. No patients with grade 4 toxicities were identified. Three grade 3 non-hematological toxicities were observed. All hematological toxicities were grade 2 .
Surgical resection. Hepatectomy procedures that were planned before neoadjuvant chemotherapy was administered were performed in all 16 patients who received the neoadjuvant chemotherapy. The interval between neoadjuvant chemotherapy and delayed surgery ranged from 30 to 114 days (median: 53 days). Operative procedures included major hepatectomy in 13 patients and minor hepatectomy in 36 patients. Complications during the postresection hospital stay occurred in $10(20 \%)$ patients. Intra-abdominal sepsis $(n=5)$ was the most common complication, followed by wound infection $(n=4)$, biliary fistula $(n=2)$, and pneumonia $(n=1)$. The incidence of postoperative morbidity was $13 \%$ (2 of 16 patients) in patients treated with neoadjuvant chemotherapy compared with $24 \%$ ( 8 of 33 patients) in patients treated 
Table III. Factors associated with MRP2 expression in 46 tumor specimens.

\begin{tabular}{|c|c|c|c|}
\hline \multirow[b]{2}{*}{ Variable } & \multicolumn{2}{|c|}{ No. of patients } & \multirow[b]{2}{*}{ P-value } \\
\hline & Non-overexpression of MRP2 & Overexpression of MRP2 & \\
\hline Age & & & 0.568 \\
\hline$\leq 70$ & 13 & 12 & \\
\hline$>70$ & 9 & 12 & \\
\hline Sex & & & $>0.999$ \\
\hline Male & 18 & 20 & \\
\hline Female & 4 & 4 & \\
\hline Neoadjuvant chemotherapy & & & 0.521 \\
\hline Absent & 17 & 16 & \\
\hline Present & 5 & 8 & \\
\hline Liver cirrhosis & & & 0.763 \\
\hline Absent & 7 & 9 & \\
\hline Present & 15 & 15 & \\
\hline Tumor size $(\mathrm{cm})$ & & & 0.388 \\
\hline$\leq 3$ & 14 & 12 & \\
\hline$>3$ & 8 & 12 & \\
\hline Number of hepatic tumors & & & 0.080 \\
\hline Solitary & 16 & 11 & \\
\hline Multiple & 6 & 13 & \\
\hline Edmondson-Steiner grade & & & 0.507 \\
\hline I & 4 & 4 & \\
\hline II & 17 & 16 & \\
\hline III & 1 & 4 & \\
\hline pT classification & & & 0.625 \\
\hline pT1 & 13 & 11 & \\
\hline pT2 & 8 & 10 & \\
\hline pT3 & 1 & 3 & \\
\hline Vascular invasion & & & $>0.999$ \\
\hline Absent & 18 & 20 & \\
\hline Present & 4 & 4 & \\
\hline
\end{tabular}

MRP2, multidrug resistance-associated protein 2; pT, pathologic T classification.

without neoadjuvant chemotherapy $(\mathrm{P}=0.464)$. There were no in-hospital deaths in the current series.

Tumor necrosis in resected specimens of 16 patients treated with cisplatin. The median percentage of histologically verified tumor necrosis was $81 \%$ (range: $0-100 \%$ ). Complete tumor necrosis (no evidence of vivid tumor) was found in 3 patients [1 patient with PR (partial response) and 2 patients with SD (stable disease)], whereas no evidence of tumor necrosis was observed in 3 patients. Tumor size on CT images prior to neoadjuvant chemotherapy did not correlate with tumor necrosis of the resected specimens $(\mathrm{P}=0.706$; Fig. $2 \mathrm{~A})$. The dose of hepatic arterial infusion of cisplatin did not correlate with tumor necrosis of the resected specimens $(\mathrm{P}=0.555$; Fig. 2B). The median percentage of tumor necrosis was $99.4 \%$ (range: $73.6-100 \%$ ) in 4 patients with PR, whereas it was $54.9 \%$ (range: $0-100 \%$ ) in 12 patients with SD. There were no apparent differences in the percentage of tumor necrosis between 2 groups (PR vs. SD) according to the RECIST criteria by Mann-Whitney $\mathrm{U}$ test $(\mathrm{P}=0.138)$.

Immunohistochemical analysis of MRP2 expression. Three tissue samples with complete tumor necrosis were excluded from immunohistochemical analysis for MRP2. In the remaining 46 tissue samples, overexpression of MRP2 was detected in 24/46 (52\%) of tumor specimens (Table III). 


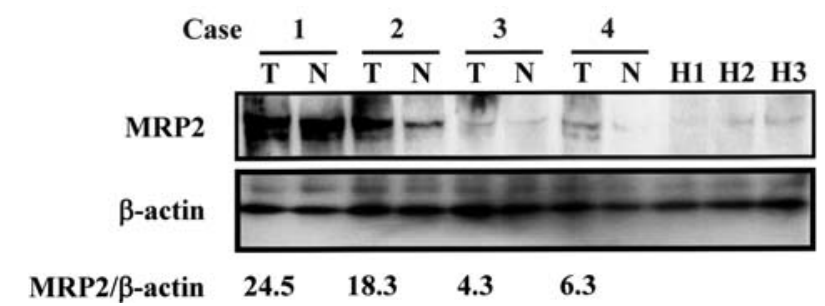

Figure 3. Western blot analysis for multidrug resistance-associated protein 2 (MRP2) levels. Based on immunohistochemical analysis, case 1 and 2 show overexpression of MRP2, whereas case 3 and 4 show non-overexpression of MRP2. The band intensities of tumor samples tested by Western blot analysis show a close correlation with the results of immunohistochemical analysis. MRP2 expression is faint in all healthy liver tissues. T; tumor, $\mathrm{N}$; non-tumor, H1-3; healthy liver.

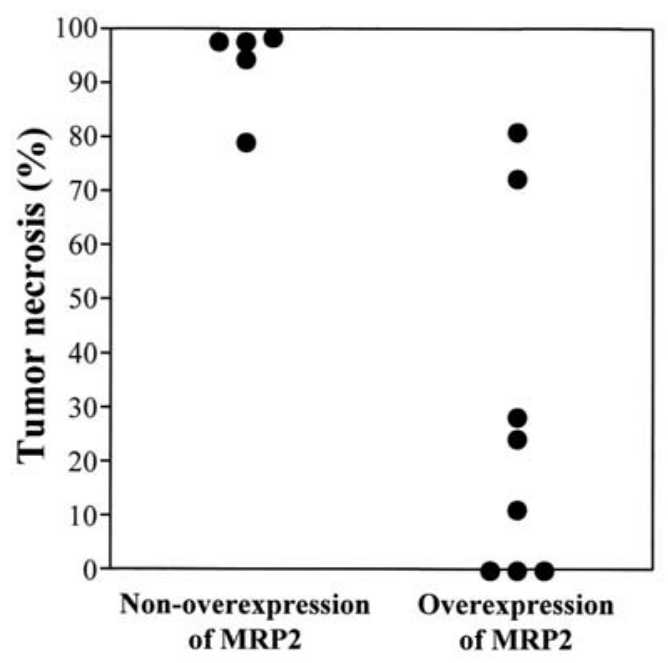

Figure 4. The extent of tumor necrosis in relation to the level of multidrug resistance-associated protein 2 (MRP2) expression. The percentage of tumor necrosis is significantly lower in tumor specimens with overexpression of MRP2 (median percentage of necrosis, 19\%) than in tumor specimens with non-overexpression of MRP2 (median percentage of necrosis, 99\%; $\mathrm{P}=0.003$ ).

Loss-of-expression of MRP2 was found in 2/46 (4\%) of tumor specimens; unchanged expression of MRP2 was observed in 20/46 (44\%) of tumor specimens; and thus 22 tumor specimens were categorized as specimens with non-overexpression of MRP2.

Western blot analyses of MRP2 expression levels. To confirm the results of the immunohistochemical analysis for MRP2, we performed Western blot analysis on 5 randomly selected tumor samples with overexpression of MRP2, 5 tumor samples with non-overexpression of MRP2, and 3 tissue samples from healthy liver. Fig. 3 shows the representative results of Western blotting for MRP2 of selected tissue samples. MRP2 expression was faint in all healthy liver tissues tested by Western blot analysis, whereas the band intensities of tumor samples showed a close correlation with the results of immunohistochemical analysis.

Factors associated with MRP2 expression in 46 tumor specimens. Overexpression of MRP2 in tumor specimens was not associated with tested clinical-pathologic factors including neoadjuvant chemotherapy (Table III). Overexpression of MRP2 was detected in 16/33 (48\%) of tumor specimens from patients treated without neoadjuvant chemotherapy, suggesting that nearly half of the patients with HCC have intrinsic overexpression of MRP2.

MRP2 expression and tumor necrosis in 13 tumor specimens containing vivid tumor from patients treated with cisplatin. We identified 8 tumor specimens with overexpression of MRP2 out of 13 tumor specimens containing vivid tumor from 16 patients treated with cisplatin-based neoadjuvant chemotherapy (Table III). Tumor specimens with overexpression of MRP2 showed a lower percentage of tumor necrosis (median percentage of necrosis, 19\%) compared to tumor specimens with non-overexpression of MRP2 (median percentage of necrosis, 99\%; $\mathrm{P}=0.003$; Fig. 4).

\section{Discussion}

The activity of $\mathrm{ABC}$ transporters is one of the major causes of resistance to chemotherapy in patients with HCC (23). Of several identified ABC transporters, MRP2 is the principal cisplatin transporter (7-9). There is a paucity of clinical data regarding any relationship between MRP2 expression and tumor necrosis in patients treated with cisplatin-based neoadjuvant chemotherapy prior to hepatectomy for HCC. This prompted us to conduct the current study. This is the first study to demonstrate that MRP2 overexpression correlates with a lower percentage of tumor necrosis in tumors from patients treated with cisplatin-based neoadjuvant chemotherapy for HCC and thus expression of MRP2 determines the efficacy of cisplatin-based chemotherapy in patients with HCC.

Cisplatin is a widely used chemotherapeutic agent in the treatment of HCC (24). The fine-powder formulation of cisplatin has 3 times higher concentration compared to conventional formulations of cisplatin (25). Yoshikawa et al (25) reported that hepatic arterial infusion of a fine-powder formulation of cisplatin had a high therapeutic efficacy with a response rate of $33.8 \%$. In the current study, we confirmed the results of Yoshikawa et al (25). Severe adverse effects (grade 3, liver dysfunction) were observed in 3 patients but they were manageable and transient. Planned hepatectomy procedures were performed in all 16 patients treated with neoadjuvant chemotherapy without any increase in postoperative complications. Thus, neoadjuvant chemotherapy with a fine-powder formulation of cisplatin is well tolerated and does not impair planned hepatectomy procedures.

We observed high tumor necrosis in the tumor specimens of patients who were treated with cisplatin-based neoadjuvant chemotherapy for HCC. In contrast, there were no apparent differences in the percentage of tumor necrosis between patients with PR and patients with SD according to RECIST criteria. In fact, complete tumor necrosis (no evidence of vivid tumor) was found in 2 patients with SD. Forner et al (26) have the questioned the reliability of RECIST criteria, which are based on the evaluation of unidimensional tumor measurements and disregard the extent of necrosis in solid liver tumors. Forner et al (26) recommended the evaluation 
of tumor response based on measurements of the reduction in viable tumor burden as determined by dynamic imaging studies. Michaelis and Ratain (27) also suggested that RECIST criteria have limited value in the assessment of tumor response after chemotherapy because some anti-cancer agents have cytostatic, rather than cytotoxic properties, so that shrinkage of the tumor after cytostatic chemotherapy is not expected. Further investigation is required to develop effective criteria for the assessment of tumor response after chemotherapy.

MRP2 is a major transporter of conjugated bilirubin and bile salts into the bile canaliculi $(5,6)$. In the current study, half of the tumor samples showed MRP2 overexpression, irrespective of administration of cisplatin (Table III). Since HCCs often produce bile, the intrinsic expression of MRP2 in HCC may partly explain their low sensitivity to some MRP2-dependent anti-cancer agents including cisplatin, doxorubicin, etoposide, and vincristine $(7,28)$. Bonin et al documented that MRP2 mRNA expression level significantly increases in HCC compared to non-neoplastic liver tissue (14). In addition, Zollner et al using Western blot analysis found that MRP2 protein expression was elevated in all 4 HCC samples examined (13). In the current study, overexpression of MRP2 correlated with a lower percentage of tumor necrosis in patients treated with cisplatin-based neoadjuvant chemotherapy for $\mathrm{HCC}$, whereas tumor size or dose of cisplatin did not. Collectively, these above findings suggest that expression of MRP2 determines the efficacy of cisplatin-based chemotherapy in patients with HCC.

Various classic compounds that inhibit MRP2 activity in vitro such as MK-571 or cyclosporin A have been proposed to be used to increase antitumor therapeutic effectiveness $(29,30)$. The intrinsic toxicity of MRP2 inhibitors at doses necessary for their activity and their poor specificity are the major obstacles in applying them in vivo (8). In attempt to develop alternative, less toxic, and more efficient therapy, Meterna et al specifically inhibited MRP2 protein expression by 2 anti-MRP 2 hammerhead ribozymes. This gene therapeutic approach may be applicable to overcome cisplatin resistance in tumor cells (8). Folmer et al demonstrated that tumors resulting from MRP2-overexpressing subcutaneously grown hepatoma cells, regressed in size upon antisense MRP2 expression in combination with vincristine (28). Wakamatsu et al reported that co-treatment with cisplatin and both glycyrrhizin and lamivudine inhibited the cisplatin efflux from Huh7 HCC cell line and concluded this was because glycyrrhizin is a competitive substrate for MRP2 (9). Therefore, it appears that inhibition of MRP2 may be an approach to develop an effective therapy to overcome cisplatin resistance in patients with HCC.

In interpreting the current study, we have considered the limitations of the analysis of a small number of patients and incomplete follow-up assessment of performed chemotherapeutic treatment using RECIST criteria. In fact, we believe that these limitations do not greatly influence the results of the study because the differences among groups were too marked to have resulted from these procedural biases.

In conclusion, overexpression of MRP2 correlates with a lower percentage of tumor necrosis in patients treated with cisplatin-based neoadjuvant chemotherapy for HCC, whereas either tumor size or dose of cisplatin does not. Expression of
MRP2 determines the efficacy of cisplatin-based chemotherapy in patients with HCC.

\section{References}

1. Dean M, Rzhetsky A and Allikmets R: The human ATP-binding cassette $(A B C)$ transporter superfamily. Genome Res 11: 1156-1166, 2001.

2. Mayer R, Kartenbeck J, Büchler M, Jedlitschky G, Leier I and Keppler D: Expression of the MRP gene-encoded conjugate export pump in liver and its selective absence from the canalicular membrane in transport-deficient mutant hepatocytes. J Cell Biol 131: 137-150, 1995.

3. Keppler D and Kartenbeck J: The canalicular conjugate export pump encoded by the cmrp/cmoat gene. In: Progress in Liver Diseases. Boyer JL and Ockner RK (eds). Saunders, Philadelphia, PA, pp55-67, 1996.

4. Paulusma CC, Kool M, Bosma PJ, Scheffer GL, ter Borg F, Scheper RJ, Tytgat GN, Borst P, Baas F and Oude Elferink RP: A mutation in the human canalicular multispecific organic anion transporter gene causes the Dubin-Johnson syndrome. Hepatology 25: 1539-1542, 1997

5. Jedlitschky G, Leier I, Buchholz U, Hummel-Eisenbeiss J, Burchell B and Keppler D: ATP-dependent transport of bilirubin glucuronides by the multidrug resistance protein MRP1 and its hepatocyte canalicular isoform MRP2. Biochem J 327: 305-310, 1997.

6. Trauner M and Boyer JL: Bile salt transporters: molecular characterization, function, and regulation. Physiol Rev 83: 633-671, 2003.

7. Cui Y, König J, Buchholz JK, Spring H, Leier I and Keppler D: Drug resistance and ATP-dependent conjugate transport mediated by the apical multidrug resistance protein, MRP2, permanently expressed in human and canine cells. Mol Pharmacol 55: 929-937, 1999.

8. Materna V, Liedert B, Thomale $\mathrm{J}$ and Lage H: Protection of platinum-DNA adduct formation and reversal of cisplatin resistance by anti-MRP2 hammerhead ribozymes in human cancer cells. Int J Cancer 115: 393-402, 2005.

9. Wakamatsu T, Nakahashi Y, Hachimine D, Seki T and Okazaki K: The combination of glycyrrhizin and lamivudine can reverse the cisplatin resistance in hepatocellular carcinoma cells through inhibition of multidrug resistance-associated proteins. Int J Oncol 31: 1465-1472, 2007

10. Koike K, Kawabe T, Tanaka T, Toh S, Uchiumi T, Wada M, Akiyama S, Ono M and Kuwano M: A canalicular multispecific organic anion transporter (cMOAT) antisense cDNA enhances drug sensitivity in human hepatic cancer cells. Cancer Res 57: 5475-5479, 1997

11. Nies AT, König J, Pfannschmidt M, Klar E, Hofmann WJ and Keppler D: Expression of the multidrug resistance proteins MRP2 and MRP3 in human hepatocellular carcinoma. Int J Cancer 94: 492-499, 2001.

12. Vander Borght S, Libbrecht L, Blokzijl H, Faber KN, Moshage H, Aerts R, Van Steenbergen W, Jansen PL, Desmet VJ and Roskams TA: Diagnostic and pathogenetic implications of the expression of hepatic transporters in focal lesions occurring in normal liver. J Pathol 207: 471-482, 2005.

13. Zollner G, Wagner M, Fickert P, Silbert D, Fuchsbichler A, Zatloukal K, Denk H and Trauner M: Hepatobiliary transporter expression in human hepatocellular carcinoma. Liver Int 25: 367-379, 2005.

14. Bonin S, Pascolo L, Crocé LS, Stanta G and Tiribelli C: Gene expression of $\mathrm{ABC}$ proteins in hepatocellular carcinoma, perineoplastic tissue, and liver diseases. Mol Med 8: 318-325, 2002.

15. Cruz PV, Wakai T, Shirai Y, Yokoyama N and Hatakeyama K: Loss of carcinoembryonic antigen-related cell adhesion molecule 1 expression is an adverse prognostic factor in hepatocellular carcinoma. Cancer 104: 354-360, 2005.

16. Wakai T, Shirai Y, Sakata J, Kaneko K, Cruz PV, Akazawa K and Hatakeyama K: Anatomic resection independently improves long-term survival in patients with T1-T2 hepatocellular carcinoma. Ann Surg Oncol 14: 1356-1365, 2007.

17. Korita PV, Wakai T, Shirai Y, Matsuda Y, Sakata J, Cui X, Ajioka Y and Hatakeyama K: Overexpression of osteopontin independently correlates with vascular invasion and poor prognosis in patients with hepatocellular carcinoma. Hum Pathol 39: 1777-1783, 2008. 
18. Therasse P, Arbuck SG, Eisenhauer EA, Wanders J, Kaplan RS, Rubinstein L, Verweij J, Van Glabbeke M, van Oosterom AT, Christian MC and Gwyther SG: New guidelines to evaluate the response to treatment in solid tumors. European Organization for Research and Treatment of Cancer, National Cancer Institute of the United States, National Cancer Institute of Canada. J Natl Cancer Inst 92: 205-216, 2000.

19. Cancer Therapy Evaluation Program. Common Terminology Criteria for Adverse Events. Version 4.0. National Cancer Institute. Available from URL: http://ctep.cancer.gov/ protocolDevelopment/electronic_applications/ctc.htm.

20. Sobin LH and Wittekind C: TNM Classification of Malignant Tumours. 6th edition. UICC, Wiley-Liss, New York, pp81-83, 2002.

21. Taylor M, Forster J, Langer B, Taylor BR, Greig PD and Mahut C: A study of prognostic factors for hepatic resection for colorectal metastases. Am J Surg 73: 467-471, 1997.

22. Edmondson HA and Steiner PE: Primary carcinoma of the liver: a study of 100 cases among 48,900 necropsies. Cancer 7: 462-503, 1954.

23. Le Vee M, Jigorel E, Glaise D, Gripon P, Guguen-Guillouzo C and Fardel O: Functional expression of sinusoidal and canalicular hepatic drug transporters in the differentiated human hepatoma HepaRG cell line. Eur J Pharm Sci 28: 109-117, 2006.

24. Carr BI: Hepatocellular carcinoma: current management and future trends. Gastroenterology 127 (Suppl 1): S218-S224, 2004.
25. Yoshikawa M, Ono N, Yodono H, Ichida T and Nakamura H: Phase II study of hepatic arterial infusion of a fine-powder formulation of cisplatin for advanced hepatocellular carcinoma. Hepatol Res 38: 474-483, 2008.

26. Forner A, Ayuso C, Varela M, Rimola J, Hessheimer AJ, de Lope CR, Reig M, Bianchi L, Llovet JM and Bruix J: Evaluation of tumor response after locoregional therapies in hepatocellular carcinoma: are response evaluation criteria in solid tumors reliable? Cancer 115: 616-623, 2009.

27. Michaelis LC and Ratain MJ: Measuring response in a postRECIST world: from black and white to shades of grey. Nat Rev Cancer 6: 409-414, 2006.

28. Folmer Y, Schneider M, Blum HE and Hafkemeyer P: Reversal of drug resistance of hepatocellular carcinoma cells by adenoviral delivery of anti-ABCC2 antisense constructs. Cancer Gene Ther 14: 875-884, 2007.

29. Leier I, Jedlitschky G, Buchholz U, Cole SP, Deeley RG and Keppler D: The MRP gene encodes an ATP-dependent export pump for leukotriene C4 and structurally related conjugates. J Biol Chem 269: 27807-27810, 1994.

30. Chen ZS, Kawabe T, Ono M, Aoki S, Sumizawa T, Furukawa T, Uchiumi T, Wada M, Kuwano M and Akiyama SI: Effect of multidrug resistance-reversing agents on transporting activity of human canalicular multispecific organic anion transporter. Mol Pharmacol 56: 1219-1228, 1999. 